\title{
Controlling the intermediate conductance states in RRAM devices for synaptic applications
}

\author{
H. García*, O. G. Ossorio, S. Dueñas, and H. Castán \\ Department of Electricity and Electronics, University of Valladolid, Paseo de Belén 15, \\ 47011 Valladolid (Spain) \\ *corresponding author e-mail: hecgar@ele.uva.es
}

\section{Keywords:}

Resistive switching memory (RRAM)

Multilevel storage

Synaptic device

\section{ABSTRACT}

RRAM devices are promising candidates to implement artificial synaptic devices for their use in neuromorphic systems, due to their high number or reachable conductance levels. The capacitors used in this work $\left(\mathrm{TiN} / \mathrm{Ti} / \mathrm{HfO}_{2} / \mathrm{W}\right)$ show resistive switching behavior and reachable intermediate conductance states. We can control the conductance states by applying voltage pulses to the top electrode. Different approaches to control the synaptic weight have been studied: applying pulses with different amplitudes changes the synaptic weight variation in an exponential way and applying pulses with different lengths changes the synaptic weight in a linear weight. We can control the conductance values when applying depression pulses, but the potentiation characteristic is not linear, as for other synaptic devices, as PRAMs. Applying other voltage signals to the structure, as voltage ramps, can improve the potentiation characteristic. 


\section{INTRODUCTION}

Neuromorphic computing systems are an alternative architecture to solve the von Neumann bottleneck: the physical separation between the memory unit and the central process unit increases latency and power consumption [1-3]. The way the data is processed and the memory operation are the main differences between the human brain and the modern processors. The human brain and other biological systems have inspired new neural network hardware architectures, where synapses, responsible for the connection of the enormous number of neurons, are a key element. Different attempts have been carried out to emulate the performance of the biological synapses by using emerging memory devices, as phase-change RAM (PRAM) [4,5], magnetic RAM (MRAM) [6] and resistive switching RAM (RRAM) [7,8]. The latter devices have also been studied to implement biological neurons $[8,9]$.

RRAM devices have been mainly investigated to implement non-volatile memories. These memories use two different resistance states (a high resistance state (HRS) and a low resistance state (LRS)) due to a conductive filament (CF) formation between the metal electrodes [10]. However, a high number of reachable conductance levels is a required property to implement analog synapses, and these devices actually show continuously variable conductance values [11]. RRAM devices are a promising approach for artificial synapses due to its fast speed $(\sim \mathrm{ns})$, ultra-low power consumption $(\sim \mathrm{nW})$ and scalability to the nanometer regime. Both conductive bridge RAM [12] and valence change memories [13] have been investigated for this purpose. However, there are still some issues about using RRAM devices as synapses that should be solved: the linearity of the resistance change between set and reset values, reliability problems and the inherently abrupt nature of the CF formation during the set process. We can try to solve the latter issue as the filament can broaden in an incremental way once it has been formed.

The objective of this work is studying different ways for controlling the intermediate conductance states in RRAM devices for synaptic applications. We have carried out our experiments using hafnium oxide based metal-insulator-metal (MIM) capacitors to implement the RRAM devices. An analysis of several ways to control the intermediate conductance states is presented. We have achieved the control of the conductance states by applying voltage pulses to the top electrode. For the depression characteristic, we have 
proved the intermediate states can be properly controlled in several ways, such as changing the voltage pulse length, amplitude and the initial maximum voltage applied to the samples. In the case of the potentiation characteristic, the control is not linear due to the abrupt set characteristic in the oxide-based RRAM devices. The use of voltage ramps instead of voltage pulses can improve the characteristic.

\section{EXPERIMENT SECTION}

The devices used in this work are metal-insulator-metal TiN/Ti/10-nm $\mathrm{HfO}_{2} / \mathrm{W}$ capacitors. The hafnium oxide $\left(\mathrm{HfO}_{2}\right)$ dielectric was deposited by atomic layer deposition

at $225{ }^{\circ} \mathrm{C}$. The precursors used were tetrakis(dimethylamido)hafnium (TDMAH) and water, as hafnium and oxygen precursors respectively. The bottom electrode consists of a $200 \mathrm{~nm} \mathrm{~W}$ layer, and the top electrode consists of a stack of a $200 \mathrm{~nm}$ TiN layer and a $10 \mathrm{~nm}$ Ti layer.

An HP 4155B Semiconductor Parameter Analyzer was used to perform the currentvoltage (I-V) measurements. In order to perform the pulsed measurements, we used an Agilent 33500B Series waveform generator to apply the voltage pulses. The current measurement equipment must be isolated from the waveform generator, so we used a relay system controlled by a Keithley 6517A electrometer. The pulse amplitude applied to the RRAM capacitor must be independent of its conductance value, so we have added a buffer at the waveform generator output using an operational amplifier with a very low output resistance and very high slew-rate to be able to apply short pulses without distortion. Fig. 1 shows the measurement set-up. The equipment were computer controlled.

\section{RESULTS AND DISCUSSION}

Fig. 2(a) shows the I-V characteristics when increasing the maximum voltage value from one loop to the next one. In a previous work [14] we found the resistive switching mechanism in our devices was valence change memory effect, so the CFs are due to oxygen vacancy clusters. The device clearly shows intermediate conductance states. 


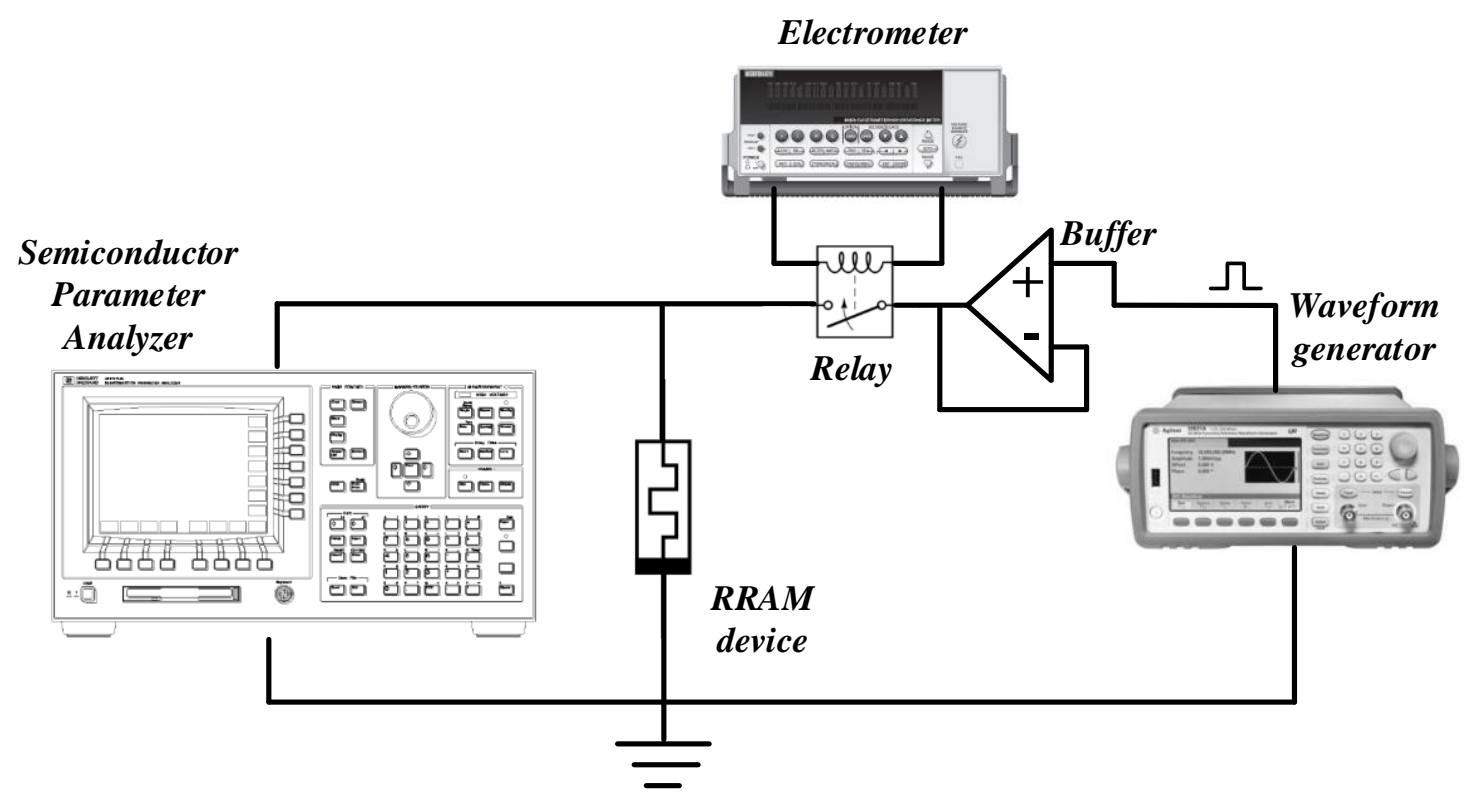

Fig. 1. Pulsed measurement set-up.

Simulations demonstrated the resistance value has a strong relationship with the maximum voltage and with the time that it is applied [15]. The only difference between consecutive pulses is the maximum voltage applied, which is responsible for the shape and thickness of the CF. Fig. 2(b) shows the conductance values for the different maximum top voltages. The voltage has to reach a minimum value before the conductivity value changes. We have achieved the control of the intermediate conductance values by applying different maximum top voltages, but increasing the maximum voltage value is a difficult way to control the intermediate states as a small change in the value or time it is applied can cause a great change in the conductivity value. This is due to the abrupt nature of the CF formation and remove process.

The spike-based neuromorphic computing require voltage (or current) pulses to modify the resistance of the RRAM cells, which is known as synaptic weight in the neuroscience. We first studied the depression characteristic, represented in Fig. 3. The sample was first carried out to the LRS by applying a maximum top voltage of $+0.8 \mathrm{~V}$. We performed three different measurements varying the pulse length and keeping the pulse amplitude constant $(-0.8 \mathrm{~V})$. The conductance values were obtained using a voltage of $-0.1 \mathrm{~V}$, a low enough value to prevent the modification of the resistivity state due to the measurement process. We can observe a continuum of intermediate conductance values properly controlled by the applied pulses. As the pulse length decreases, we need a larger number 

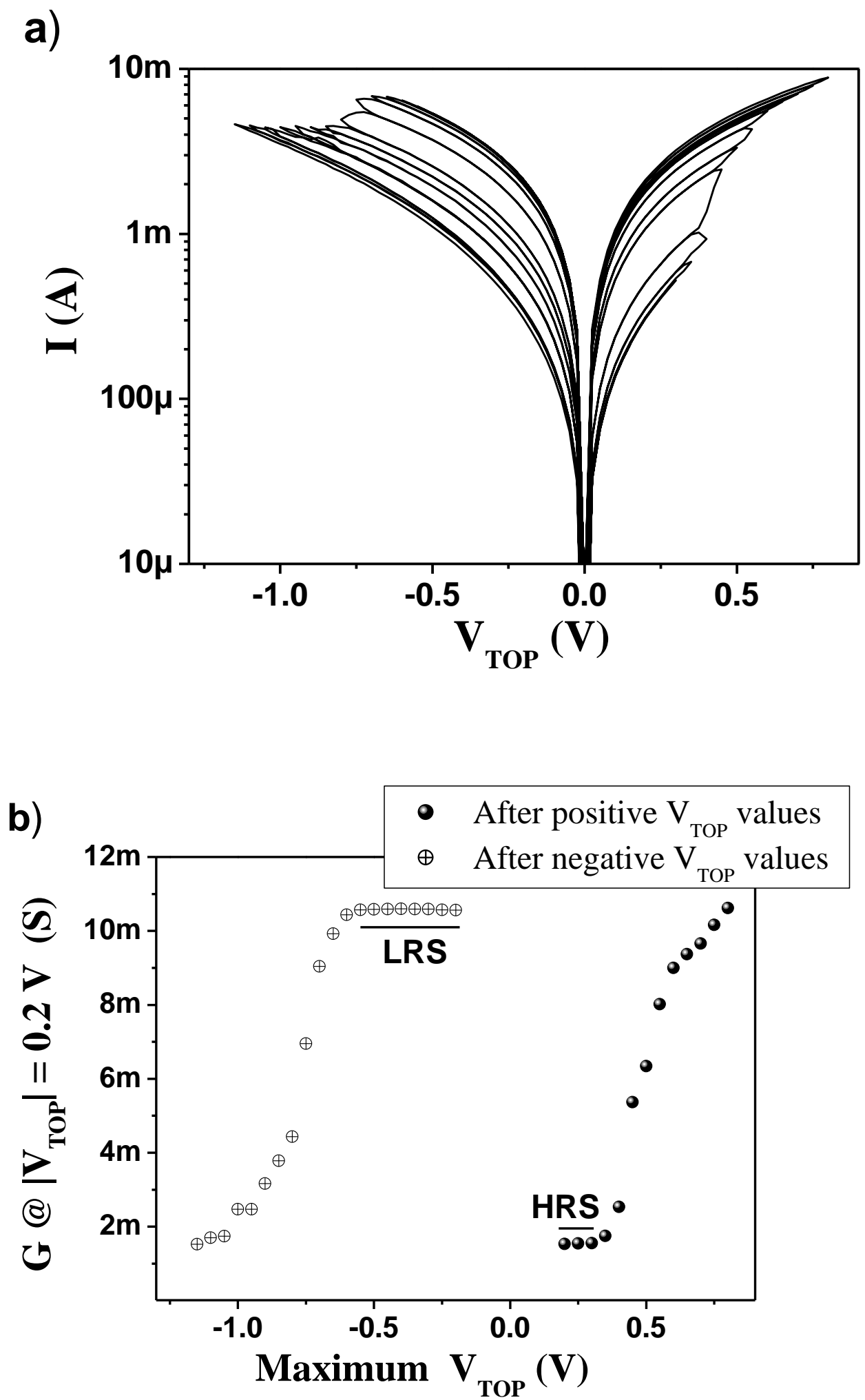

Fig. 2. I-V characteristics measured increasing the maximum voltage values applied to the top of the structure from one loop to the next one (a) and intermediate conductance values obtained (b). 
of voltage pulses to dissolve the conductive filament. When the conductance reaches a value of $\approx 5 \mathrm{mS}$, the dispersion in the values is noticeable. It was found in similar devices that when the CF is partially disrupted, there is a dispersion in the resistivity values, although this result was obtained by limiting the maximum voltage [7]. Applying consecutive depression pulses, we finally obtain a gap between the tip of the $\mathrm{CF}$ and the electrode.

The relationship between conductance and the number of pulses is linear for higher conductance values, which indicates the thickness of the CF can be modulated. However, two different slopes are observed in Fig. 3: a lower slope value for conductance values higher than $\approx 8 \mathrm{mS}$, and a higher value for lower conductance values. Two different $\mathrm{CF}$ narrowing rates are obtained in the measurements. The two different slopes are represented in Fig. 4 for each pulse length. The conductance variation $v s$ pulse length relation is linear for each conductance region: the depression pulse length is able to control the synaptic weight variation in a linear way.

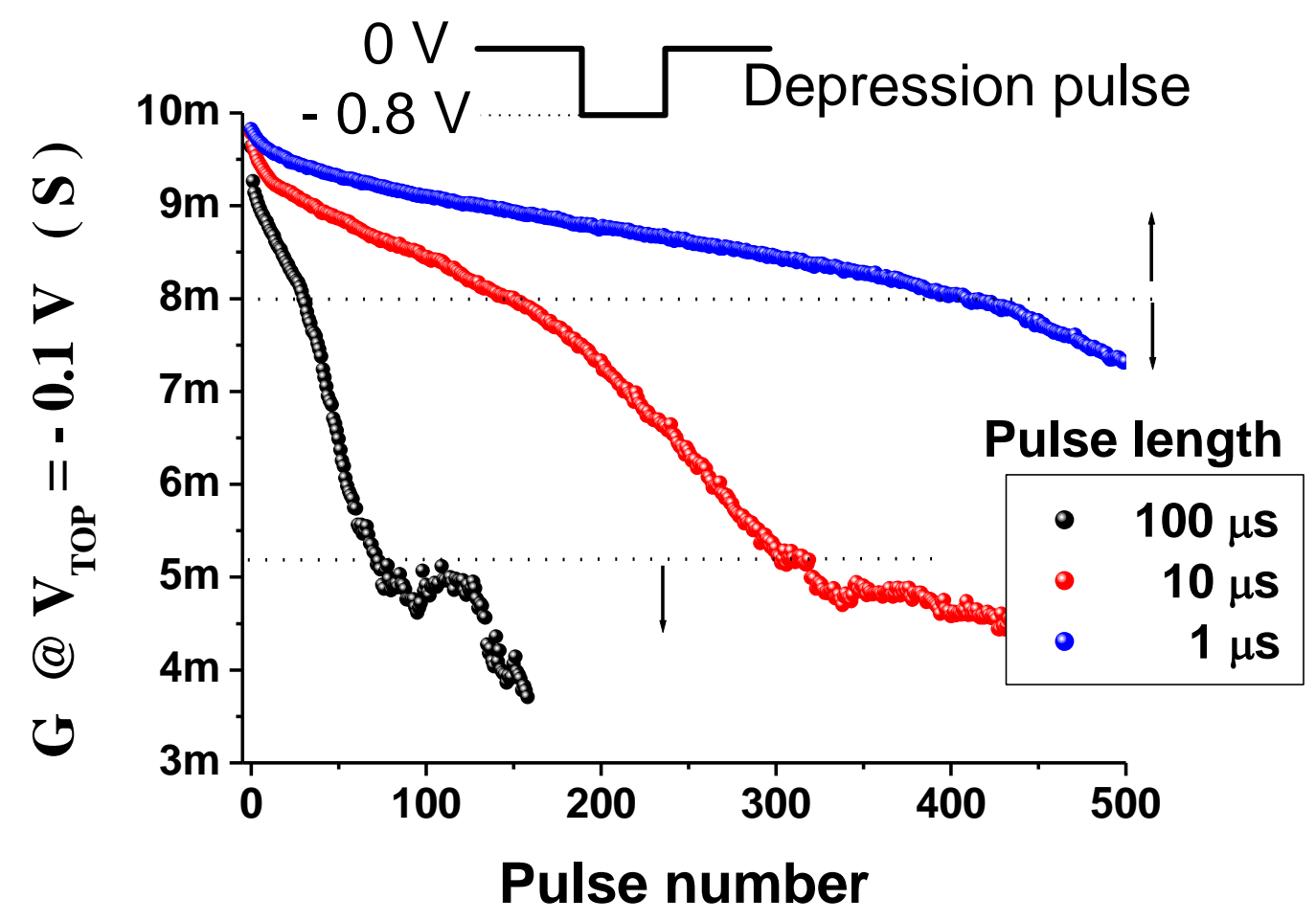

Fig. 3. Depression characteristic for identical initial state and three different depression pulse length. 


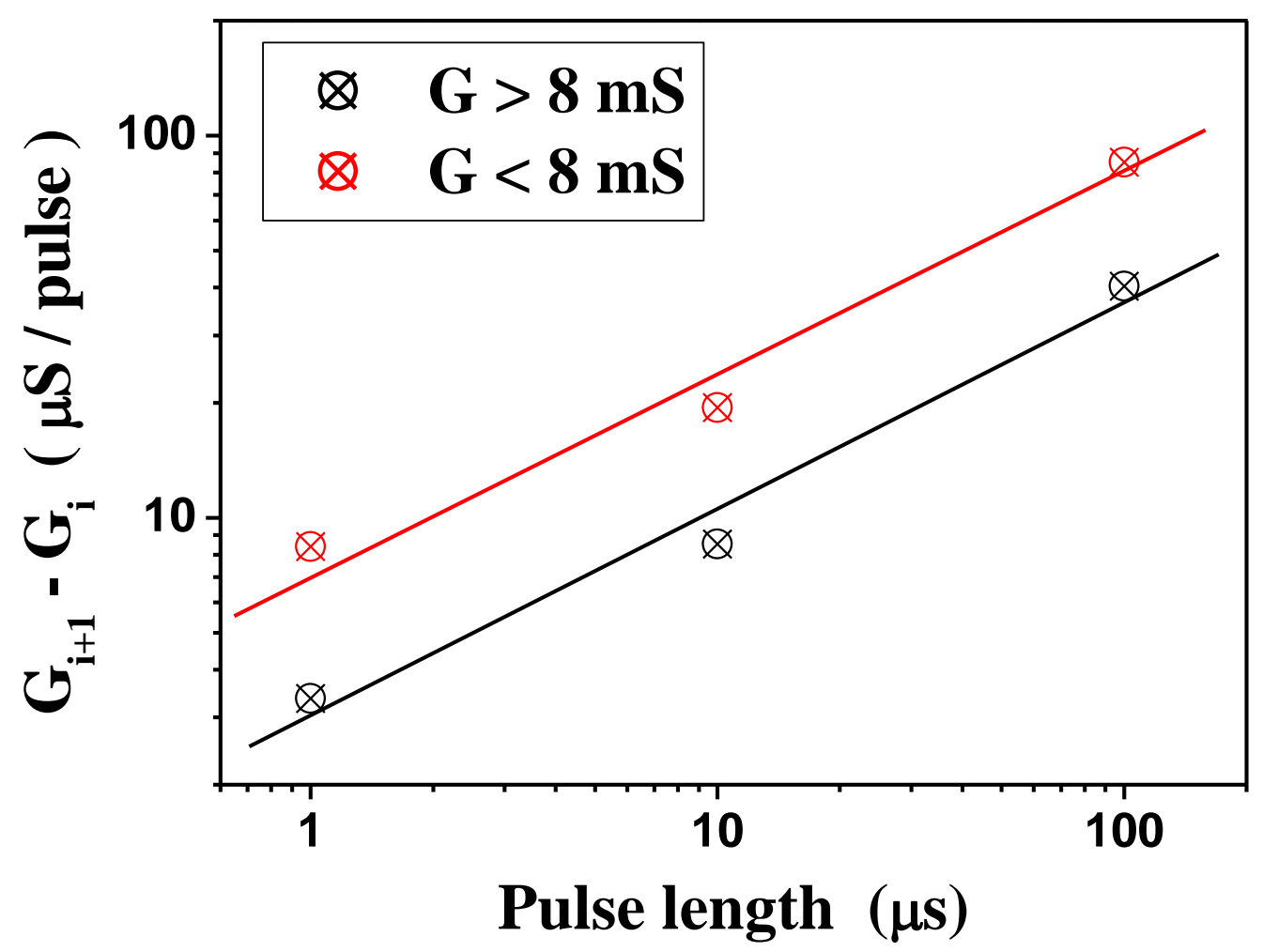

Fig. 4. Conductance variation $v s$ depression pulse length shows a linear trend for each conductance region.

We have also studied the effect of the initial maximum top voltage in the control of the conductance values. Fig. 5 shows in a semi-log plot the depression characteristic for different initial maximum voltages. The initial conductance value decreases as the initial voltage decreases, because the initial thickness of the $\mathrm{CF}$ is smaller, and a different number of voltage pulses are required to obtain the same conductance value. However, the most interest fact is once the same conductance value has been reached, a different number of identical depression pulses has to be applied to obtain identical conductivity variation (an example is represented in Fig. 5). Higher initial voltages strengthen the LRS, probably due to the formation of different filamentary paths between both electrodes. We are able to control the synaptic weight variation after the application of identical pulses by adjusting the initial maximum voltage. 


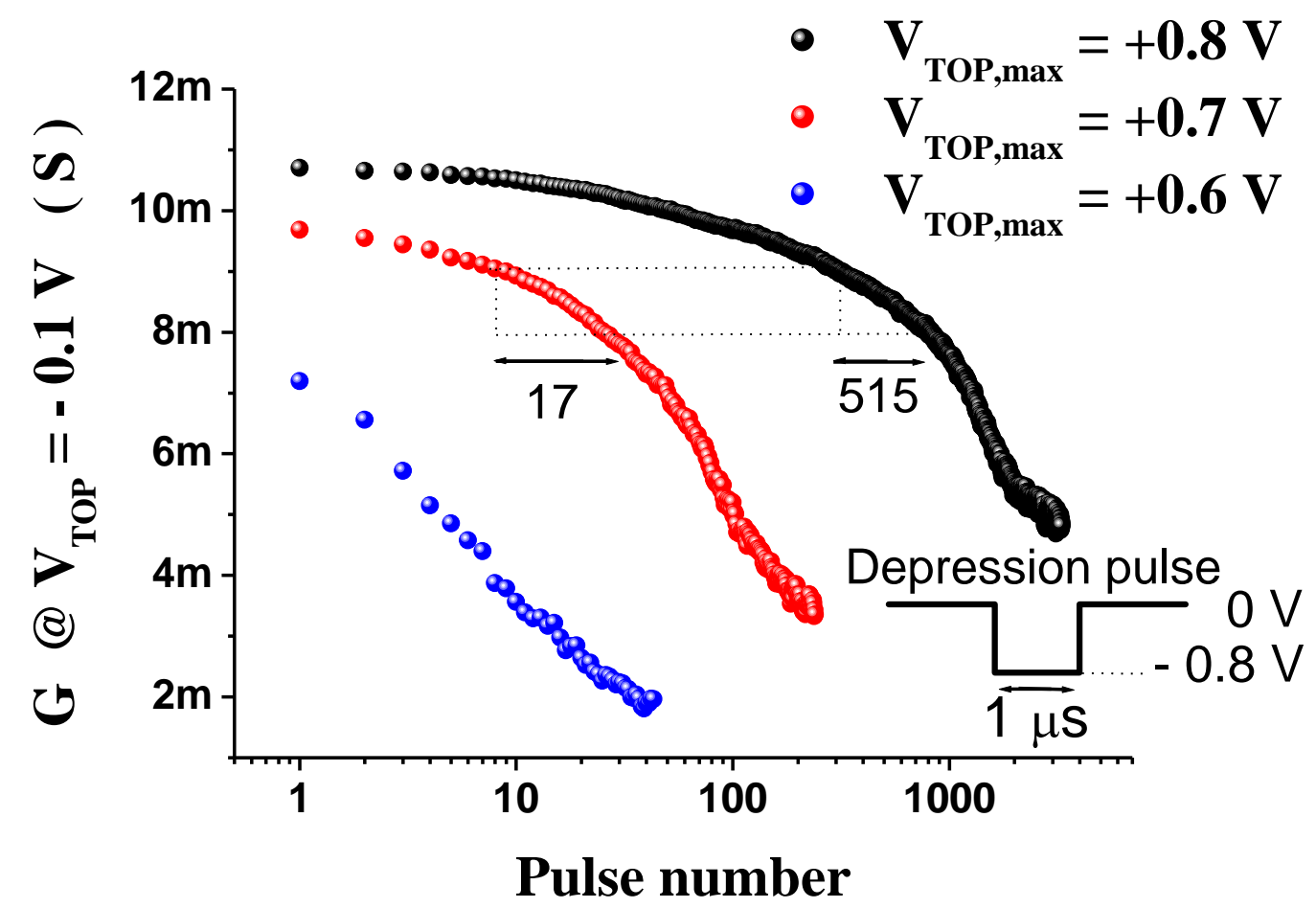

Fig. 5. Depression characteristic for different initial maximum voltages and identical depression pulses.

Another way to control the synaptic weight variation is changing the programming pulse amplitude. A general trend that has been observed is the programming pulses length decreases when increasing the pulse amplitude for the same resistivity value target [16]. Fig. 6 shows the depression characteristic for three different measurements, now varying the pulse amplitude while keeping the pulse length constant $(1 \mu \mathrm{s})$. As the pulse amplitude increases, a lower number of pulses are needed to decrease the conductance value. However, unlike the pulse length variation case, the relation of $\Delta \mathrm{G} v s$ pulse amplitude is not linear, as we can see in Fig. 7. In this case, we can observe an exponential relation. This is due to the ion hopping process through the potential well of the oxide matrix, because the drift velocity of the ion migration depends exponentially with the average electric field inside the oxide [17]. So higher conductance variations are obtained adjusting the pulse amplitude, and more accurate variations are obtained adjusting the pulse length. However, it has been found in terms of both faster and energy efficient reset programming, its preferable to use larger amplitudes and shorter pulse lengths [16]. 


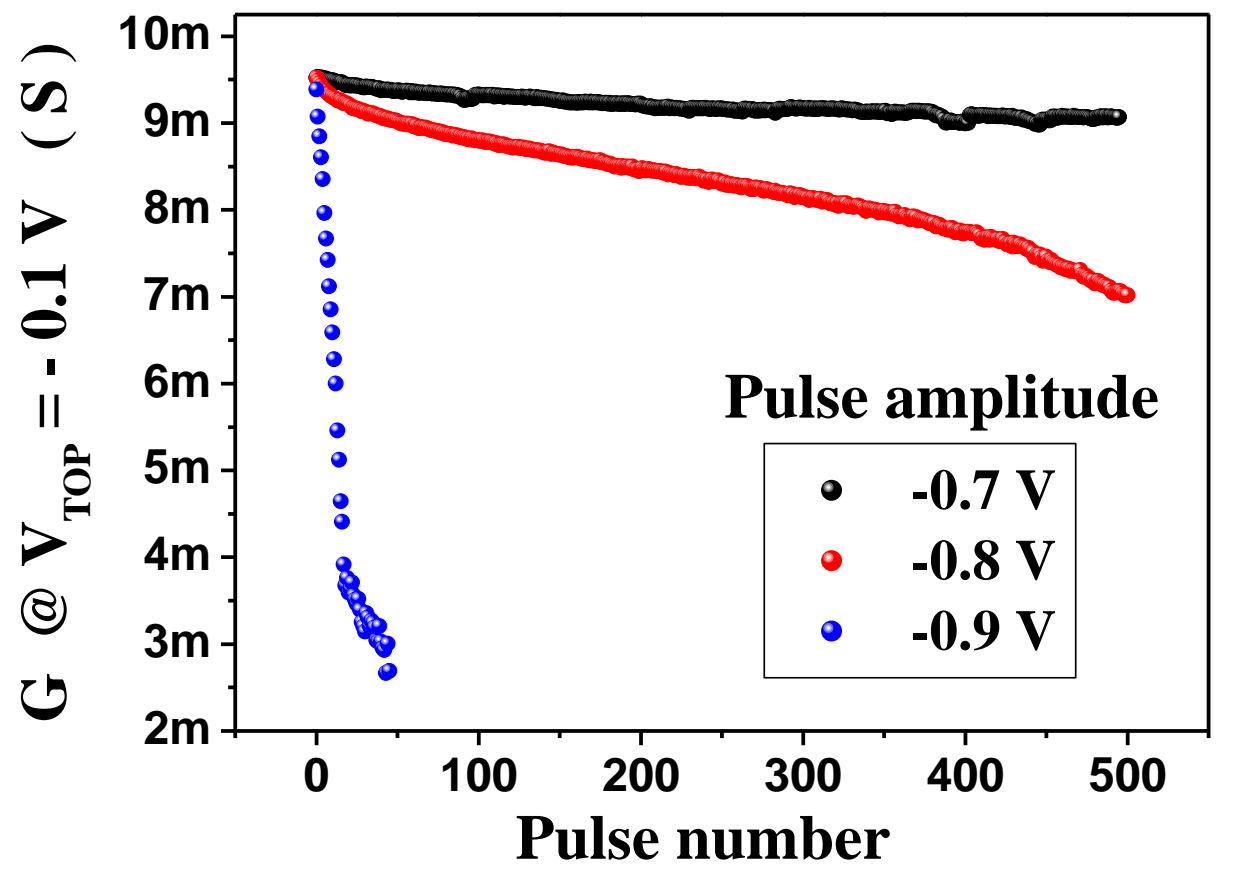

Fig. 6. Depression characteristic for identical initial state and three different depression pulse amplitude.

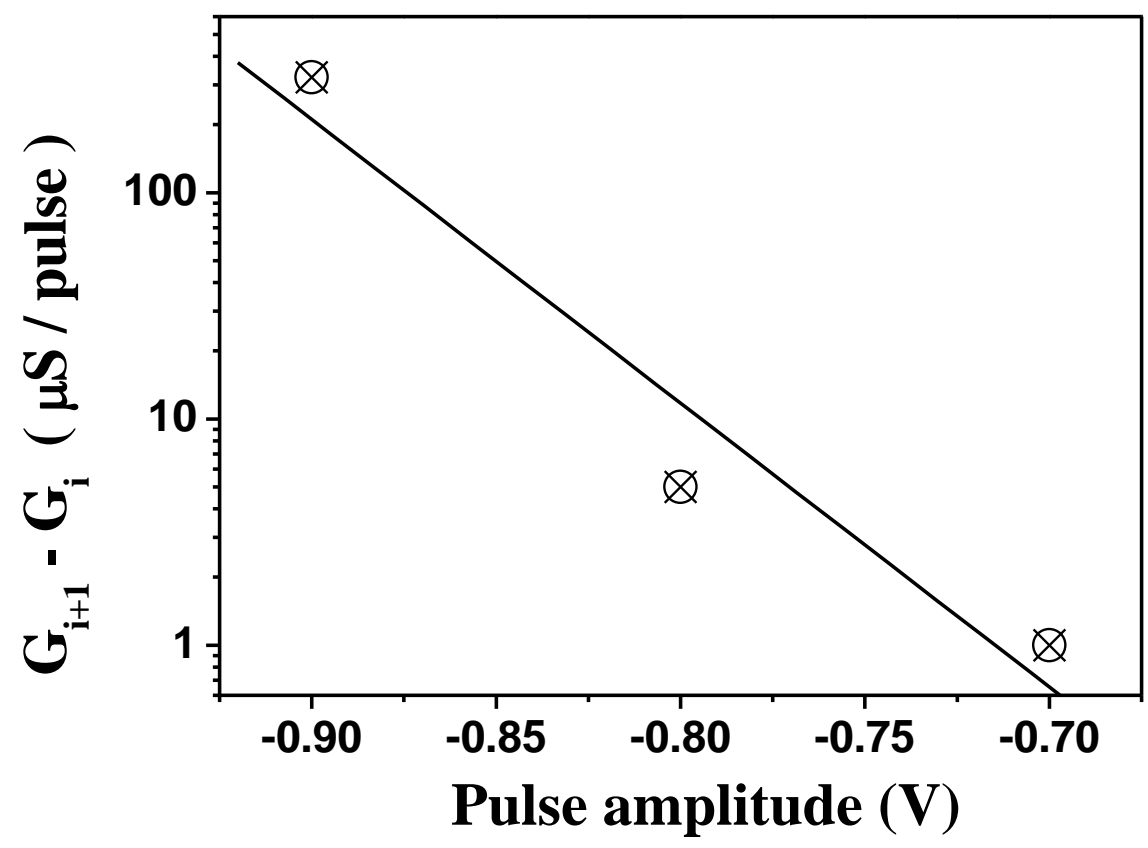

Fig. 7. Conductance variation $v s$ depression pulse amplitude shows an exponential trend. 
The potentiation characteristics are quite different. Fig. 8 shows the depression and potentiation characteristics measured consecutively. First, the sample was carried out to the LRS applying a maximum voltage of $+0.7 \mathrm{~V}$. The characteristics of the applied pulses are shown in Fig. 8 caption. It is difficult to obtain symmetric characteristics in metaloxide RRAM devices because of the different set and reset transitions. The reset transition has been used to tune the synaptic weight [18]. However, the abrupt set transition inhibit tuning the synaptic weight, as in phase change devices [2]. Decreasing even more the pulse length or the pulse amplitude is not a choice, as it has been found that the probability for a set event decreases as the pulse amplitude or length decreases [19]. Some approaches were used to solve this problem. Employing two RRAM devices (2R synapse) with opposite weight contribution can improve the performance [20]. Some authors reported a gradual set switching applying a proper reset pulse after the potentiation pulse [19], and linearly increasing the amplitude of the potentiation pulses have also used for this purpose [21]. However, this has disadvantages in terms of peripheral circuit complexity.

We have studied the use of voltage ramps instead of linearly increasing the amplitude of the pulses. Using this kind of signal, the voltage amplitude linearly increases during each ramp application, instead of after consecutive pulses. This has the advantage of using a single signal for potentiation and depression. Fig. 9 represents the depression and potentiation characteristic using voltage ramps instead of voltage pulses. Although the characteristic remains asymmetric, the synaptic weight variation when applying potentiation ramps is much less abrupt. So applying this kind of voltage signals can assist better linear and symmetric characteristics.

\section{CONCLUSIONS}

Hafnium oxide-based MIM capacitors present resistive switching effect, and show intermediate conductance states, a necessary property for their use as synaptic devices. These intermediate states can be reached by changing the maximum voltage applied in a sweep voltage mode. However, this is not an efficient manner for reaching the conductance values. We can control the conductance values in a more accurate way by applying voltage pulses to the top electrode. The synaptic weight can be precisely 


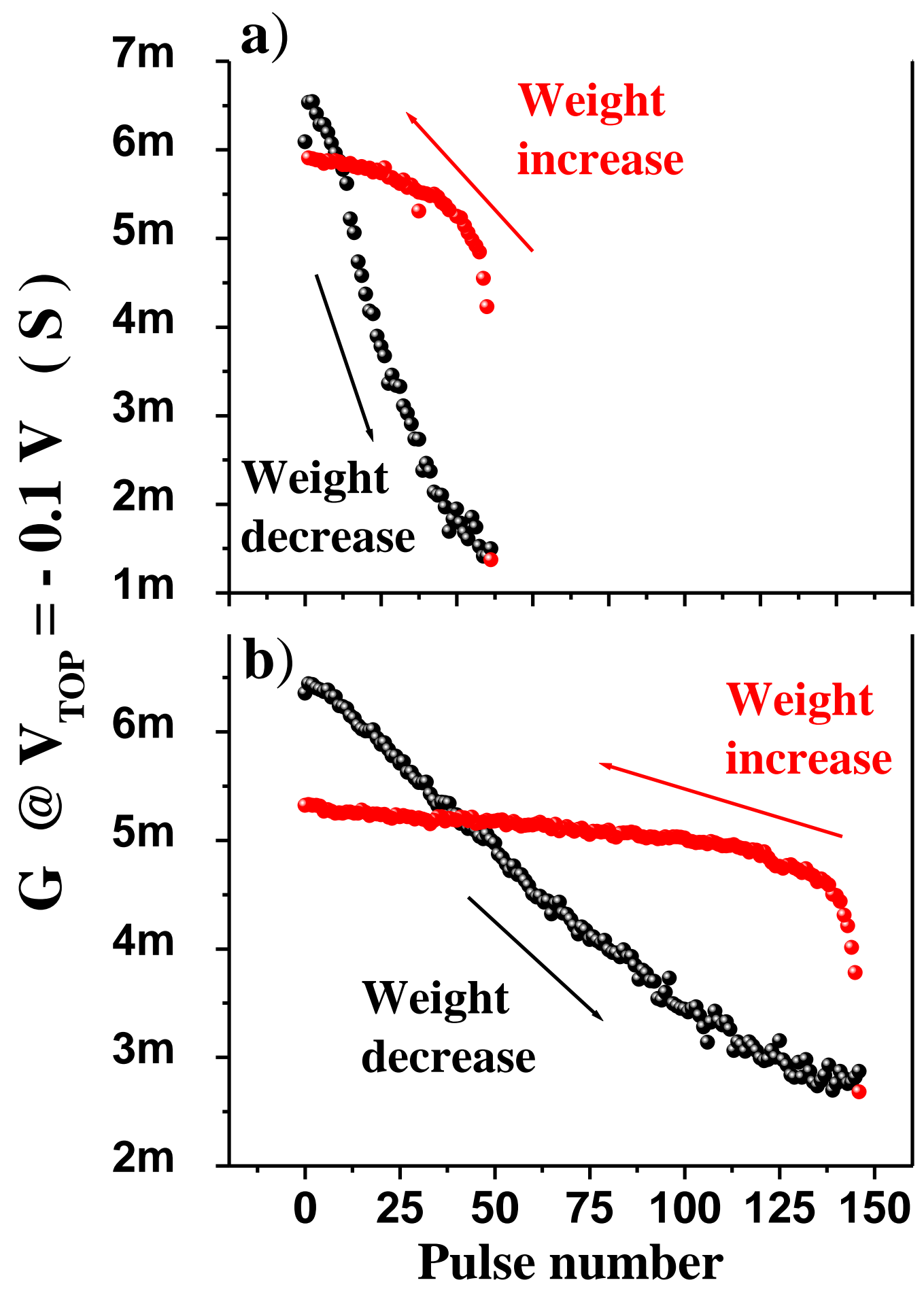

Fig. 8. Potentiation and depression characteristic for identical initial state and using 100 ns potentiation and depression pulses. (a) Depression pulse amplitude $=-1 \mathrm{~V}$, potentiation pulse amplitude $=+0.7 \mathrm{~V}$. (b) Depression pulse amplitude $=-0.9 \mathrm{~V}$, potentiation pulse amplitude $=+0.6 \mathrm{~V}$. 


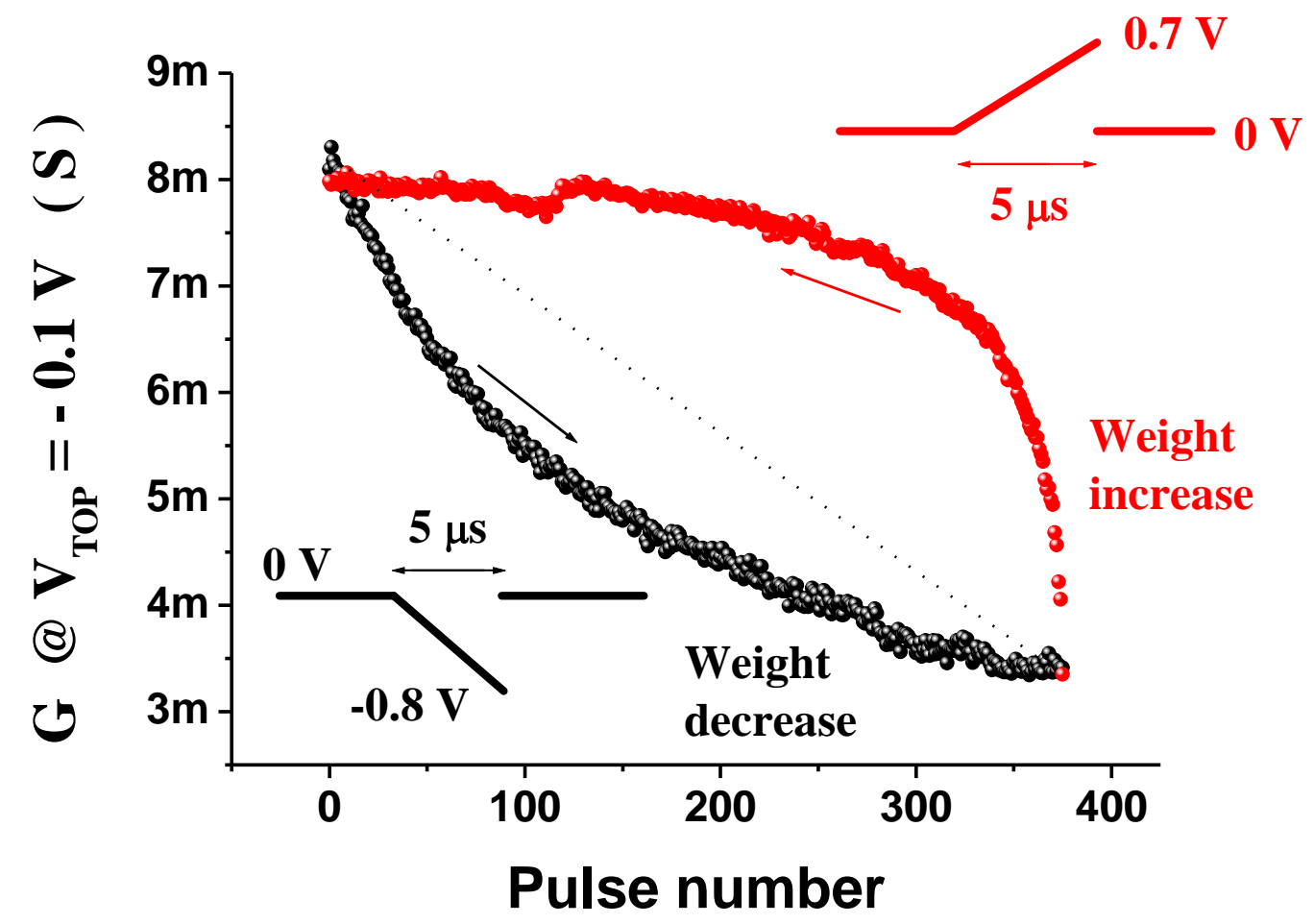

Fig. 9. Potentiation and depression characteristic using voltage ramps instead of voltage pulses.

controlled when applying depression pulses, especially for the control of the conductive filament thickness. In this case, a pretty linear characteristic has been obtained. The synaptic weight variation can be performed by several ways, for instance changing the pulse length or the pulse amplitude. A linear conductance variation is obtained if we increase the pulse length. In the second option, we obtain an exponential conductance variation, due to the ion hopping process through the potential well of the oxide matrix, responsible for the formation of the conductive filament. The synaptic weight variation can also be modified for identical voltage pulses, by adjusting the initial maximum voltage applied to the device: higher initial maximum voltages firm up the high conductance state. The conductance $v s$ pulse number characteristic is not linear for the potentiation characteristic, due to the abrupt set transition observed in this kind of structures. However, we can improve the linearity using other kind of signals instead of voltage pulses, for instance voltage ramps. 


\section{ACKNOWLEDGMENT}

This work was funded by the Spanish Ministry of Economy and Competitiveness and the FEDER program through project TEC2017-84321-C4-2-R. This work has made use of the Spanish ICTS Network MICRONANOFABS.

Authors would like to acknowledge Prof. Campabadal group from the Institute of Microelectronics of Barcelona (IMB-CNM-CSIC, Spain) for providing the samples of this study.

\section{REFERENCES}

[1] C. Sung, H. Hwang, and I. K. Yoo, J. Appl. Phys. 124 (2018) 151903.

[2] D. Ielmini, Microelectron. Eng. 190 (2018) 44-53.

[3] Y. Chen, H. Li, C. Wu, C. Song, S. Li, C. Min, H-P. Cheng, W. Wen, and X. Liu, Integration-VLSI J. 61 (2018) 49-61.

[4] G. W. Burr, R. M. Shelby, S. Sidler, C. Nolfo, J. Jang, I. Boybat, R. S. Shenoy, P. Narayanan, K. Virwani, E. U. Giacometti, B. N. Kurdi, and H. Hwang, IEEE Trans. Electron Devices 62 (2015) 3498-3507.

[5] O. Bichler, M. Suri, D. Querlioz, D. Vuillaume, B. de Salvo, and C. Gamrat, IEEE Trans. Electron Devices 59 (2012) 2206-2214.

[6] A. F. Vincent, J. Larroque. N. Locatelli, N. B. Romdhane, O. Bichler, C. Gamrat, W. S. Zhao, J.-O. Klein, S. Galdin-Retailleau, and D. Querlioz, IEEE Trans. Biomed. Circuits Syst 9 (2015), 166-174.

[7] M. Pedro, J. M. Martinez, M. B. Gonzalez, R. Rodriguez, F. Campabadal, M. Nafria, and X. Aymerich, Microelectron. Eng. 178 (2017) 89-92.

[8] J. Woo, D. Lee, Y. Koo, and H. Hwang, Microelectron. Eng. 182 (2017) 42-45.

[9] T. Tuma, A. Pantazi, M. Le Gallo, A. Sebastian, and E. Eleftheriou, Nat. Nanotechnol. 11 (2016) 693-699.

[10] T-C. Chang, K-C. Chang, T-M. Tsai, T-J. Chu and S. M. Sze, Mater. Today 19 (2016) 254-264. 
[11] H. Castán, S. Dueñas, H. García, O. G. Ossorio, L. A. Domínguez, B. Sahelices, E. Miranda, M. B. González, and F. Campabadal, J. Appl. Phys. 124 (2018) 152101.

[12] S. Yu, and S. P. Wong, in International Electron Device Meeting Technical Digest, Ed IEEE, 2010, p.22.1.

[13] S. Yu, Y. Wu, R. Jeyasingh, D. G. Kuzum, and H.S.P. Hwong, IEEE Trans. Electron Devices 58 (2011) 2729-2737.

[14] G. González-Cordero, M. B. González, H. García, F. Campabadal, S. Dueñas, H. Castán, F. Jiménez-Molinos, and J. B. Roldán, Microelectron. Eng. 178 (2017) 2629.

[15] Y. Li, M. Zhang, S. Long, J. Teng, Q. Liu, H. Lv, E. Miranda, J. Suñé, and M. Liu, Sci. Rep. 7 (2017) 11204.

[16] S. Yu, Y. Wu, and H.-S. P. Wong, Appl. Phys. Lett. 98 (2011) 103514.

[17] N. F. Mott, and R. W. Gurney, Electronic Processes in Ionic Crystals, Clarendon, Oxford, 1948.

[18] S. Yu, B. Gao, Z. Fang, H. Yu, J. Kang, and H.-S. P. Wong, Adv. Mater. 25 (2013) 1774-1779.

[19] K. Moon, S. Lim, J. Park, C. Sung, S. Oh, J. Woo, J. Lee, and H. Hwang, Faraday Discuss. 213 (2019) 421-451.

[20] G. W. Burr, R. M. Shelby, C. di Nolfo, R. Shenoy, P. Narayanan, K. Virwani, E. Giacometti, B. Kurdi, and H. Hwang, in IEDM Technical Digest, IEEE (2014) 29.5.

[21] J.-W. Jang, S. Park, G. W. Burr, H. Hwang, and Y.-H. Jeong, Electron Device Lett. 36 (2015) 457-459. 